\title{
Nitidine chloride inhibits proliferation and induces apoptosis in colorectal cancer cells by suppressing the ERK signaling pathway
}

\author{
HUIYUAN ZHAI ${ }^{1,2}$, SANYUAN HU $^{1}$, TONGXIANG LIU ${ }^{3}$, FENG WANG ${ }^{4}$, XIXUN WANG ${ }^{2}$, \\ GUOCHANG WU ${ }^{2}$, YIFEI ZHANG ${ }^{2}$, MINGHUA SUI ${ }^{2}$, HUANTAO LIU ${ }^{1}$ and LIXIN JIANG ${ }^{2}$ \\ ${ }^{1}$ Department of General Surgery, Qilu Hospital, Shandong University, Jinan, Shandong 250012; \\ ${ }^{2}$ Department of Abdominal Surgery, Yantai Yuhuangding Hospital, Yantai, Shandong 264000; ${ }^{3}$ Department of Emergency, \\ People's Hospital of Weifang, Weifang, Shandong 261041; ${ }^{4}$ Department of Breast Disease, \\ The Second Hospital of Shandong University, Jinan, Shandong 250033, P.R. China
}

Received February 27, 2015; Accepted December 23, 2015

DOI: $10.3892 / \mathrm{mmr} .2016 .4827$

\begin{abstract}
Nitidine chloride (NC) is a natural bioactive phytochemical alkaloid that has displayed anticancer activity in various types of cancer. However, no evidence has been reported for the direct effect of NC on CRC cell proliferation and apoptosis, and the underling mechanisms to be fully elucidated. The present study aimed to investigate the influence of $\mathrm{NC}$ on the apoptosis and proliferation of CRC cells. The viability and proliferation of CRC cells was measured by MTT assay and a $\left[{ }^{3} \mathrm{H}\right]$ thymidine uptake assay. Apoptosis was measured using a flow cytometric apoptosis assay and TUNEL staining. The expression levels of apoptotic-regulated proteins in addition to extracellular signal-regulated kinase (ERK) were measured by western blot analysis following stimulation with NC. The results indicated that $\mathrm{NC}$ inhibited the proliferation of HCT116 cells in a dose- and time-dependent manner. Additionally, apoptotic induction by $\mathrm{NC}$ treatment was confirmed. Furthermore, $\mathrm{NC}$ was demonstrated to significantly upregulate the expression of Bax, p53, cleaved caspase-3 and -9 and downregulate the expression of Bcl-2. Treatment with NC reduced the phosphorylation of ERK and by using an ERK inhibitor, U0126, the roles of NC in apoptotic induction and the inhibition of proliferation were further demonstrated. These results demonstrated that NC inhibited the proliferation
\end{abstract}

Correspondence to: Professor Lixin Jiang, Department of Abdominal Surgery, Yantai Yuhuangding Hospital, 20 Yuhuangding East Road, Yantai, Shandong 264000, P.R. China

E-mail: lixinjiangsubmit@163.com

Professor Huantao Liu, Department of General Surgery, Qilu Hospital, Shandong University, 107 Wenhua Xi Road, Jinan, Shandong 250012, P.R. China

E-mail: liuhuantaosubmit@163.com

Key words: nitidine chloride, apoptosis, proliferation, colorectal cancer cell, extracellular signal-regulated kinase and induced the apoptosis of CRC cells via the ERK signaling pathway.

\section{Introduction}

Colorectal cancer (CRC) is one of the most commonly diagnosed malignant diseases worldwide, with greater than one million new cases per year. Worldwide, it was the fourth leading cause of cancer associated-mortality in $2011(1,2)$. At present, surgical resection is the primary treatment for localized CRC. However, despite progress made in the early diagnosis and treatment of CRC which has resulted in improved prognosis for patients, the majority of patients that are not suitable for surgery present with distant metastasis at the time of diagnosis $(3,4)$. Thus, the therapeutic options for unresectable or metastatic CRC remain limited. Therefore, there is an urgent requirement for novel agents for patients with $\mathrm{CRC}$, in order to achieve improved treatment outcomes.

Recently, natural products have gained extensive attention as novel anti-cancer therapeutic agents for their relatively few side effects (5). Nitidine chloride (NC; Fig. 1A) is a natural bioactive phytochemical alkaloid that was first derived from the root of Zanthoxylum nitidum (Roxb). Previous studies have demonstrated that NC has anti-oxidant, anti-fungal, anti-inflammatory and analgesic functions $(6,7)$. In recent years, $\mathrm{NC}$ has been reported to possess anti-tumor activity in various types of cancer. $\mathrm{NC}$ has been observed to induce apoptosis and inhibit the proliferation and metastasis of renal cancer $(8,9)$. Additionally, $\mathrm{NC}$ was observed to inhibit the proliferation of breast cancer and suppress its migration and invasion via the c-Src/focal adhesion kinase signaling pathway $(10,11)$. In digestive system neoplasms, studies have reported that $\mathrm{NC}$ inhibits the proliferation of hepatocellular carcinoma (12), and suppresses angiogenesis and the growth of gastric cancer via the signal transducer and activator of transcription 3 signaling pathway (13). However, no evidence has been reported regarding the direct effect of $\mathrm{NC}$ on apoptosis and proliferation in CRC and the mechanisms of this effect.

In the present study, the effects of NC on CRC cell proliferation and apoptosis were evaluated, in addition to the underlying molecular mechanisms. 


\section{Materials and methods}

Cell lines and reagents. The HCT116 human CRC cell line was obtained from American Type Culture Collection (Manassas, VA, USA) and cells were routinely cultured in Dulbecco's modified Eagle's medium (DMEM; Invitrogen; Thermo Fisher Scientific, Inc, Waltham, MA, USA) containing $10 \%$ fetal bovine serum (FBS; Invitrogen; Thermo Fisher Scientific, Inc.), $100 \mathrm{U} / \mathrm{ml}$ penicillin, and $100 \mu \mathrm{g} / \mathrm{ml}$ streptomycin (MACGENE Biotechnology, Ltd., Beijing, China) in $5 \% \mathrm{CO}_{2}$ at $37^{\circ} \mathrm{C}$. The annexin $\mathrm{V}$-fluorescein isothiocyanate (FITC) apoptosis detection kit was purchased from BD Biosciences (Franklin Lakes, NJ, USA). A terminal deoxynucleotidyl transferase dUTP nick end labeling (TUNEL) kit was purchased from Beyotime Institute of Biotechnology (Shanghai, China). Monoclonal rabbit anti-human extracellular signal-regulated kinase (ERK)1/2 (1:1,000; cat. no. 4695), monoclonal rabbit anti-human phosphorylated (p)-ERK1/2 (1:1,000; cat. no. 4370), monoclonal rabbit anti-human $\mathrm{Bcl}-2$ (1:1,000; cat. no. 2870), monoclonal rabbit anti-human Bax (1:1,000; cat. no. 5023), monoclonal rabbit anti-human caspase-9 (1:1,000; cat. no. 9502, monoclonal rabbit anti-human caspase-3 (1:1,000; cat. no. 9665) and monoclonal rabbit anti-human p53 antibodies $(1: 1,000$; cat. no.2527) were purchased from Cell Signaling Technology, Inc. (Danvers, MA, USA). NC was purchased from Shanghai Tauto Biotech Co., Ltd. (Shanghai, China) and dissolved in dimethyl sulfoxide (DMSO; Beijing Solarbio Science \& Technology Co., Ltd., Beijing, China). U0126, which is an inhibitor of mitogen-activated protein kinase 1/2 located upstream of ERK1 and ERK2 thus regulating ERK activity, was purchased from Sigma-Aldrich (St. Louis, MO, USA).

3-(4,5-dimethylthiazol-2-yl)-2,5-diphenyltetrazolium bromide (MTT) assay of cell viability and proliferation. Cell viability and proliferation were assessed by MTT assay. HCT116 cells (5,000 cells/well) in $100 \mu \mathrm{l}$ medium were seeded into 96 -well plates. Cells were pretreated with U0126 $(10 \mu \mathrm{M})$ for $5 \mathrm{~min}$. Following stimulation with $\mathrm{NC}$ of various concentrations $(0,2.5,5,10$ and $20 \mu \mathrm{M})$ for various durations $(0,6,12$, 24 and $48 \mathrm{~h}$ ), $20 \mu \mathrm{l}$ MTT $(5 \mathrm{mg} / \mathrm{ml})$ was added to each well. Following incubation for $4 \mathrm{~h}, 100 \mu \mathrm{l}$ of dimethyl sulfoxide (DMSO) was added to each well for a further $15 \mathrm{~min}$. Finally, the absorbance values were determined using an microplate luminometer (iMark; Bio-Rad Laboratories, Inc., Hercules, CA, USA) at $490 \mathrm{~nm}$.

$\left[{ }^{3} \mathrm{H}\right]$ Thymidine uptake. HCT116 cells were cultured in DMEM with $10 \%$ FBS to $50 \%$ confluence. The cells were then cultured in serum-free DMEM for $24 \mathrm{~h}$ and treated with $\mathrm{NC}$ of various concentrations $(0,2.5,5,10,20 \mu \mathrm{M})$ for $40 \mathrm{~h}$. $\left[{ }^{3} \mathrm{H}\right]$ thymidine (final concentration $1 \mathrm{uCi} / \mathrm{ml}$; PerkinElmer, Inc., Waltham, MA, USA) was added to the media during the last $24 \mathrm{~h}$ of culture. Following washing with ice-cold phosphate-buffered saline (PBS), the cells were precipitated with ice-cold 5\% trichloroacetic acid (TCA; Sigma-Aldrich) for a minimum of $4 \mathrm{~h}$ and washed twice with ice-cold $5 \%$ TCA followed by two washes with ice-cold PBS. Subsequently, the cells were lysed with $200 \mu 10.5 \mathrm{M} \mathrm{NaOH}$ for $30 \mathrm{~min}$ at $37^{\circ} \mathrm{C}$. DNA synthesis was measured by $\left[{ }^{3} \mathrm{H}\right]$ thymidine uptake using a liquid scintillation counter (LS-6500; Beckman Coulter, Inc., Brea, CA, USA).

Analysis of apoptosis by annexin V-FITC/propidium iodide (PI) staining. Apoptosis in HCT116 cells was determined using an annexin V-FITC/PI assay. In brief, HCT116 cells were seeded into 6 -well plates at $1 \times 10^{6}$ cells/well. Following treatment with $\mathrm{NC}(0,10,20 \mu \mathrm{M})$ for $24 \mathrm{~h}$, HCT116 cells were then harvested, washed and resuspended in PBS. Apoptotic cells were determined with an annexin V-FITC apoptosis detection kit according to the manufacturer's protocol. Briefly, the cells were washed and subsequently incubated for $15 \mathrm{~min}$ at room temperature in the dark in $200 \mu 1 \mathrm{X}$ binding buffer containing $5 \mu \mathrm{l}$ annexin V-FITC and $10 \mu \mathrm{l}$ PI. Apoptosis was determined by the BD Accuri C6 Flow Cytometer (BD Biosciences) and processed using Flowjo software (version 7.6.5; Flowjo LLC, Ashland, OR, USA).

Analysis of apoptosis by TUNEL assays. The TUNEL method was used to label the apoptotic cells using a TUNEL apoptosis detection kit (Beyotime Institute of Biotechnology) according to the manufacturer's protocol. Briefly, HCT116 cells were incubated with $\mathrm{NC}(0,10,20 \mu \mathrm{M})$ for $24 \mathrm{~h}$. Following incubation, the cells were fixed with $4 \%$ formaldehyde and permeabilized with $0.1 \%$ Triton $\mathrm{X}-100$ at $4^{\circ} \mathrm{C}$ for $10 \mathrm{~min}$. The cells were then stained by the TUNEL mixture for $1 \mathrm{~h}$, followed by staining with 4,6-diamidino-2-phenylindole (DAPI) for $5 \mathrm{~min}$. The TUNEL positive cells (red staining) were photographed and evaluated qualitatively using Nikon Eclipse Ti (Nikon Corp, Tokyo, Japan) and Ultra VIEW:emoji:VOX confocal microscopes (PerkinElmer, Inc.), and images were analyzed by using Volocity software (version 6.0; PerkinElmer, Inc.).

Western blot analysis. Protein was extracted from cells using a protein lysis buffer (Beyotime Institute of Biotechnology) immediately after the end of treatment. Total cell protein concentrations were determined using a bicinchoninic acid protein assay kit (Pierce Biotechnology, Inc., Rockford, IL, USA). Equal amounts of protein $(10 \mu \mathrm{g})$ from the cell lysates were run with $12 \%$ sodium dodecyl sulfate-polyacrylamide gel electrophoresis (Bio-Rad Laboratories, Inc.). Following electrophoresis, proteins were transferred to polyvinylidene membranes (EMD Millipore, Billerica, MA, USA), blocked with $5 \%$ fat-free milk at room temperature for $1 \mathrm{~h}$, and incubated with the indicated primary antibodies overnight at $4^{\circ} \mathrm{C}$. Subsequently, the membranes were washed with Tris-buffered $0.2 \%$ saline-Tween 20 and incubated with horseradish peroxidase-conjugated goat anti-rabbit secondary antibodies (1:10,000; cat. no. ZDR-5306; Beijing Zhongshan Golden Bridge Biotechnology Co., Ltd., Beijing, China) for $1 \mathrm{~h}$ at room temperature. Immune complexes were detected with enhanced chemiluminescence reagents (EMD Millipore, Boston, MA, USA), and the blots were quantified by densitometric analysis using the Alpha Imager 2200 (Genetic Technologies, Inc., Miami, FL, USA).

Statistical analysis. The data are expressed as the mean \pm standard deviation. All of the experiments were repeated a minimum of three times. Comparisons between the group values were performed by one-way analysis of variance. Holm's t-test was 
A<smiles></smiles>

C

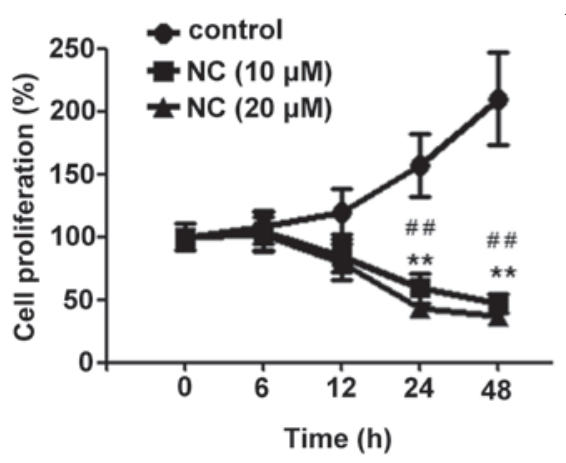

B

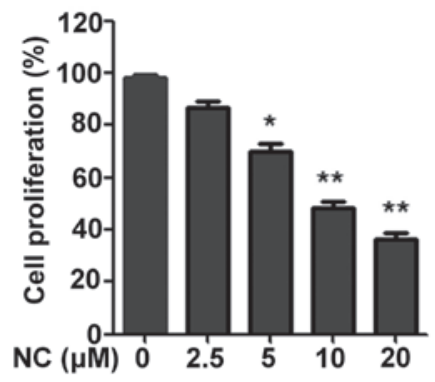

D

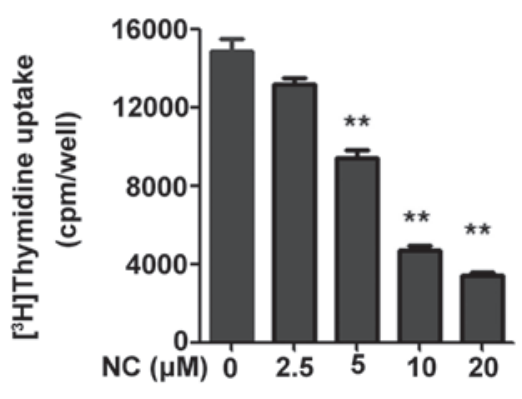

Figure 1. NC inhibits the proliferation of HCT116 cells. (A) Chemical structure of NC. (B) Following treatment with NC for $24 \mathrm{~h}(0,2.5,5,10$ and $20 \mu \mathrm{M})$, an MTT assay was used to measure the proliferation of the HCT116 cells. (C) Following treatment with 0,10 and $20 \mu \mathrm{M} \mathrm{NC}$ for different time durations $(0,6,12$, 24 and $48 \mathrm{~h}$ ), an MTT assay was used to detect the proliferation of the HCT116 cells. (D) Following treatment with NC for $40 \mathrm{~h}$ at different concentrations (0, 2.5, 5, 10 and $20 \mu \mathrm{M}), \mathrm{a}^{3} \mathrm{H}$-thymidine uptake assay was used to detect the proliferation of the HCT116 cells. The results are presented as the mean \pm standard deviation from three independent experiments. ${ }^{*} \mathrm{P}<0.05,{ }^{* *} \mathrm{P}<0.01$ vs. the control group; ${ }^{*} \mathrm{P}<0.05,{ }^{\# \#} \mathrm{P}<0.01$ vs. the cells treated with 10 and $20 \mu \mathrm{M}$ of $\mathrm{NC}$. NC, nitidine chloride; MTT, 3-(4,5-dimethylthiazol-2-yl)-2,5-diphenyltetrazolium bromide; cpm, counts per minute.

used for multiple comparisons between the groups. All statistical analysis was performed in SPSS (version 10.0; SPSS, Inc., Chicago, IL, USA) $\mathrm{P}<0.05$ was considered to indicate a statistically significant difference.

\section{Results}

NC inhibits the proliferation of CRC cells. To investigate the effect of NC on CRC cell proliferation, an MTT assay was performed. As shown in Fig. 1B and C, NC inhibited the proliferation of CRC cells in a time- and dose-dependent manner. To further assess the result, the effects of NC on CRC cell proliferation were detected by $\left[{ }^{3} \mathrm{H}\right]$ thymidine uptake. The result demonstrated that $\mathrm{NC}$ is able to significantly inhibit CRC cell proliferation over a range of concentrations $(5,10$ and $20 \mu \mathrm{M}$; Fig. 1D). Considering that the optimal inhibitory effect was observed when the NC concentration was 10 and $20 \mu \mathrm{M}$, these concentrations of $\mathrm{NC}$ were selected for the subsequent experiments. Taken together, these data suggest that NC inhibits the proliferation of CRC cells.

$N C$ induces apoptosis in CRC cells. To determine whether the NC-induced inhibition of proliferation was due to a direct effect on apoptosis in CRC cells, the cells were treated with 0,10 and $20 \mu \mathrm{M} \mathrm{NC}$ for $24 \mathrm{~h}$. As indicated by the annexin V-FITC/PI assay, NC markedly increased apoptosis in CRC cells at doses of 10 and $20 \mu \mathrm{M}$ (Fig. 2A and B). The percentage of cells undergoing apoptotic cell death increased from $6.9 \pm 1.3$ to $34.8 \pm 6.8$ and $36.9 \pm 7.2 \%$ following exposure to 10 and $20 \mu \mathrm{M} \mathrm{NC}$, respectively, for $24 \mathrm{~h}$. TUNEL was used to detect the fragmented DNA in cells undergoing apoptosis. Following NC treatment $(0,10$ and $20 \mu \mathrm{M})$ for $24 \mathrm{~h}$, the cells were stained with TUNEL and DAPI and analyzed by fluorescence microscopy. As shown in Fig. $2 \mathrm{C}$ and D, 10 and $20 \mu \mathrm{M}$ $\mathrm{NC}$ increased the proportion of apoptotic cells. The significant induction of apoptosis by NC treatment demonstrated its anti-cancer effect on CRC cells.

$N C$ induces apoptosis in CRC cells via alterations of Bcl-2 family proteins and caspase activation. To further investigate the potential mechanism of NC-induced CRC cell apoptosis, the impact of $\mathrm{NC}$ on the expression levels of Bcl-2, Bax, p53, caspase- 3 and -9 were examined. The western blotting results indicated that, following treatment with 10 and $20 \mu \mathrm{M} \mathrm{NC}$, the expression of the anti-apoptotic protein, $\mathrm{Bcl}-2$, was reduced and the pro-apoptotic protein Bax was increased. In addition, the expression levels of cleaved caspase- 3 and -9 , and p53 were upregulated (Fig. 3A and B).

$N C$ inhibits ERK phosphorylation in CRC cells. The ERK pathway serves an important role in tumor development. The inhibition of ERK pathway activation has been demonstrated to induce tumor cell apoptosis (14). To gain further insight into the association between $\mathrm{NC}$ and the proliferation and apoptosis of CRC cells, ERK signaling molecules were investigated. As presented in Fig. 4A, ERK phosphorylation was reduced following treatment with 10 and $20 \mu \mathrm{M} \mathrm{NC}$ for $24 \mathrm{~h}$, indicating that the ERK pathway may be involved in CRC cell apoptosis. The reduction was statistically significant, as quantified by densitometry (Fig. 4B). 
A

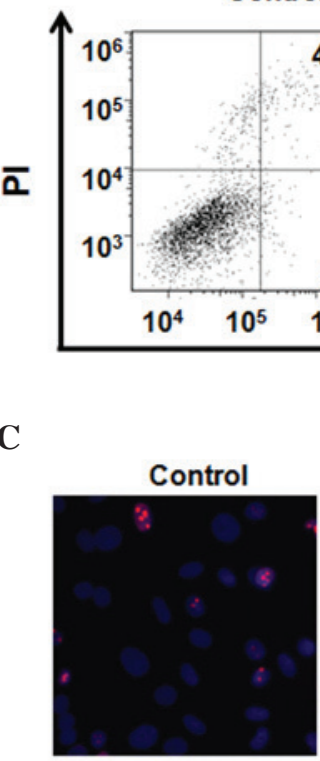

NC $(10 \mu \mathrm{M})$

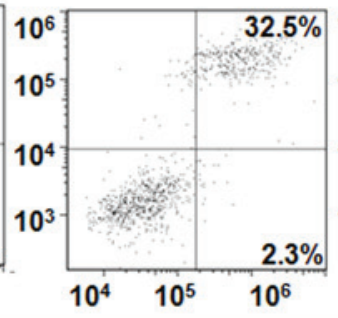

$\mathrm{NC}(20 \mu \mathrm{M})$

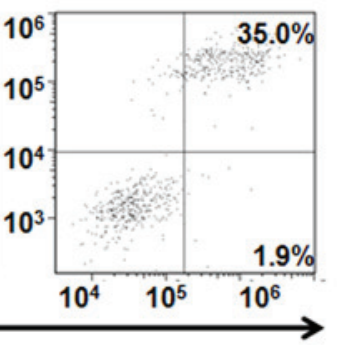

\section{Annexin V}

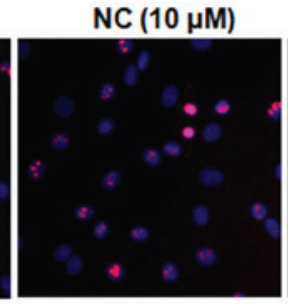

\section{$\mathrm{NC}(20 \mu \mathrm{M})$}

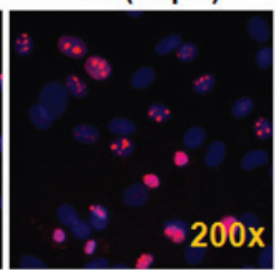

B

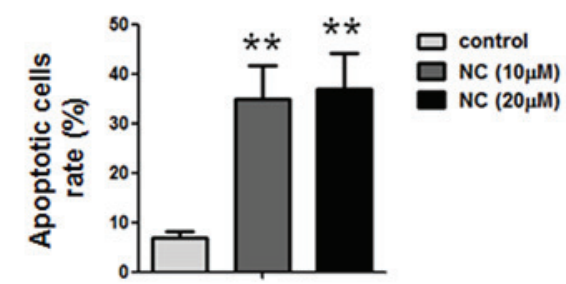

D

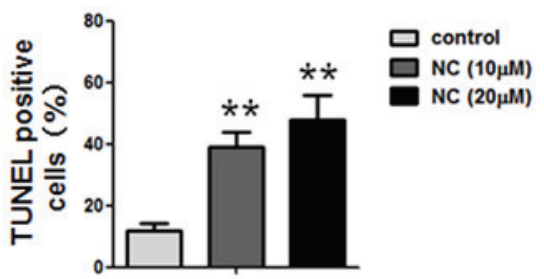

Figure 2. NC induced dose-dependent apoptosis in HCT116 cells. (A) The HCT116 cells were treated with NC $(0,10$ and $20 \mu \mathrm{M})$ for $24 \mathrm{~h}$ and then double-stained with PI and annexin V. The cells were analyzed using a flow cytometer. (B) The bar graph represents the results of three independent experiments. (C) The HCT116 cells were treated with NC $(0,10$ and $20 \mu \mathrm{M})$ for $24 \mathrm{~h}$ and then stained with DAPI and TUNEL, which labeled the 3'-OH ends of the fragmented DNA in apoptotic cells, and were viewed using a confocal microscope (magnification, x200). (D) Quantification of the percentage of TUNEL positive cells. The bar graph represents the results of three independent experiments. ${ }^{* *} \mathrm{P}<0.01$ vs. the control group. NC, nitidine chloride; PI, propidium iodide; DAPI, 4',6-diamidino-2-phenylindole; TUNEL, terminal deoxynucleotidyl transferase dUTP nick end labeling.

A

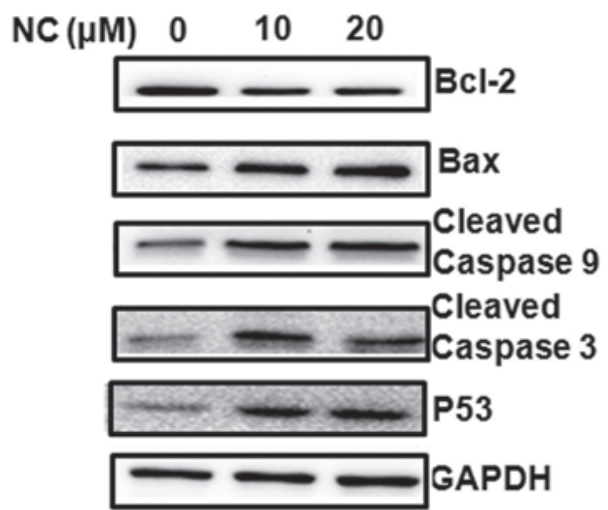

B

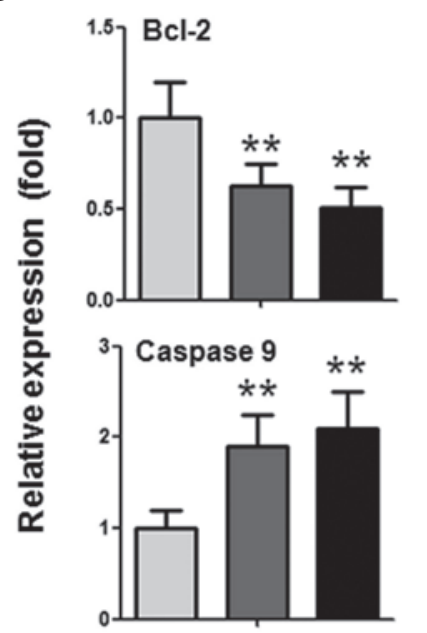

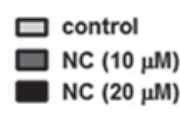

Figure 3. NC increases the expression Bax, cleaved caspase-3 and -9 and reduces the expression of Bcl-2. (A) The HCT116 cells were treated with NC $(0,10$ and $20 \mu \mathrm{M}$ ) for $24 \mathrm{~h}$, and Bax, Bcl-2, cleaved caspase-3 and -9 expression levels in cell lysates were analyzed by western blotting. (B) Quantification of the western blotting results. Data are presented as the mean \pm standard deviation from three independent experiments. ${ }^{* *} \mathrm{P}<0.01 \mathrm{vs}$. the control group. NC, nitidine chloride. 
A

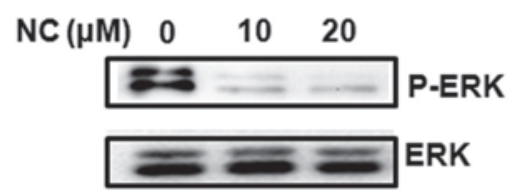

C

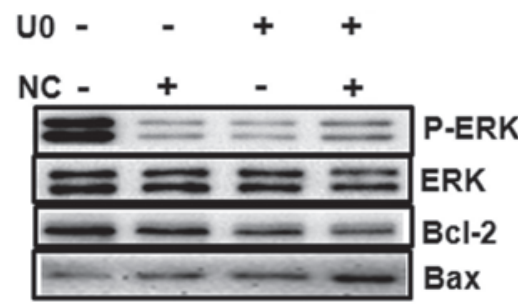

B
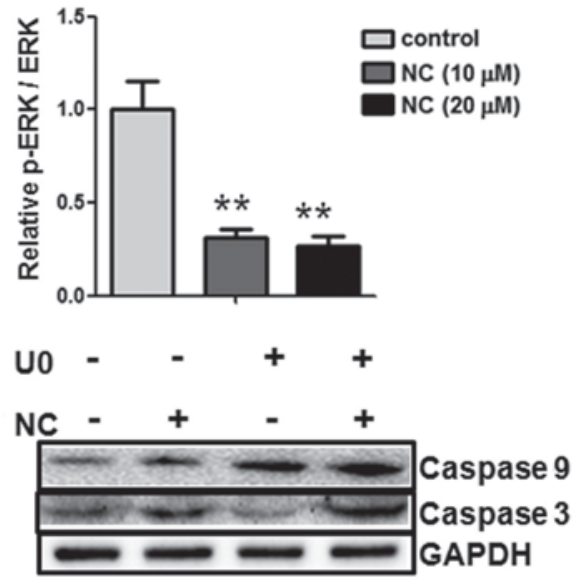

D

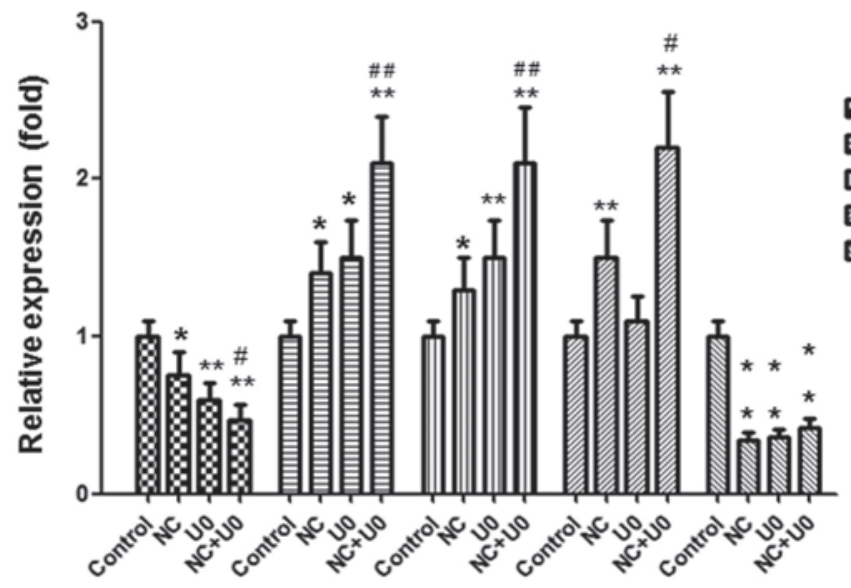

Figure 4. Pro-apoptotic effect of NC on HCT116 cells is ERK-dependent. (A) The HCT116 cells were treated with NC $(0,10$ and $20 \mu \mathrm{M})$ for $24 \mathrm{~h}$, and the levels of p-ERK1/2, ERK1/2 were analyzed by western blotting. (B) Quantification of the western blotting results. (C) The HCT116 cells were treated with $\mathrm{NC}(20 \mu \mathrm{M})$ and/or $\mathrm{U} 0126(10 \mu \mathrm{M})$ for $24 \mathrm{~h}$, the levels of p-ERK1/2, ERK1/2, Bcl-2, Bax, cleaved caspase-3 and -9 were analyzed by western blotting with GAPDH as a control. (D) Quantification of the western blotting results. Data are presented as the mean \pm standard deviation from three independent experiments."P $<0.05,{ }^{* *} \mathrm{P}<0.01$ vs. the control group. ${ }^{\#} \mathrm{P}<0.05,{ }^{\# \prime} \mathrm{P}<0.01$ vs. the cells treated with $\mathrm{NC}$. NC, nitidine chloride; ERK, extracellular-signal-regulated kinase; p, phosphorylated; GAPDH, glyceraldehyde 3-phosphate dehydrogenase; U0, U0126.
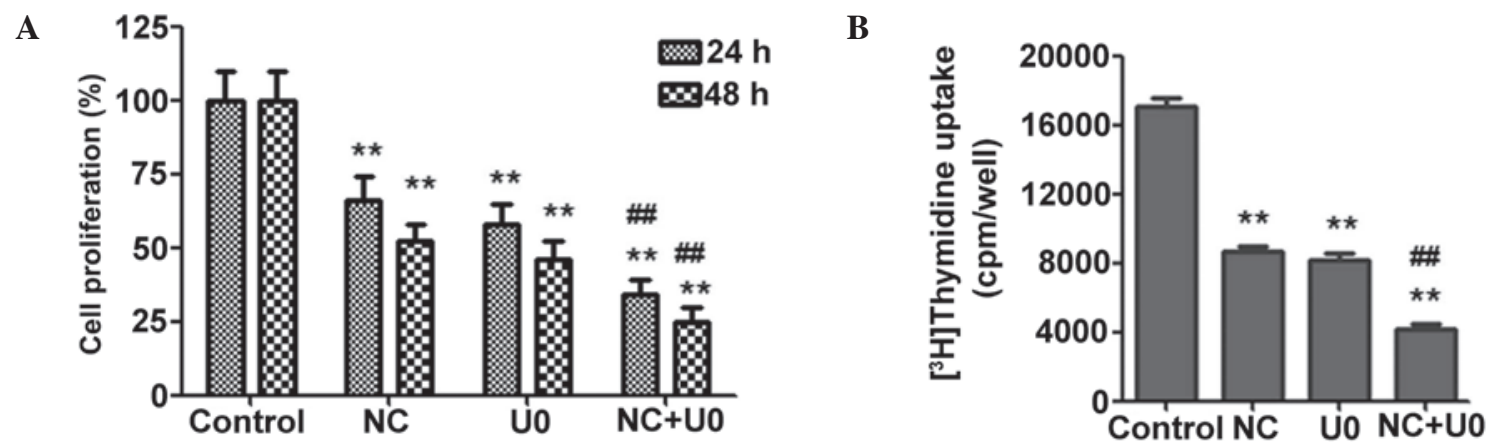

Figure 5. NC inhibition of HCT116 cell proliferation is ERK-dependent. (A) The HCT116 cells were treated with NC (20 $\mu \mathrm{M})$ and/or U0126 (10 $\mu \mathrm{M})$ for $24 \mathrm{~h}$ and $48 \mathrm{~h}$, and cell viability was measured by 3-(4,5-dimethylthiazol-2-yl)-2,5-diphenyltetrazolium bromide assay. (B) The HCT116 cells were treated with NC $(20 \mu \mathrm{M})$ and/or U0126 $(10 \mu \mathrm{M})$ for $40 \mathrm{~h}$, and cell proliferation was measured by ${ }^{3} \mathrm{H}$-thymidine uptake assay. The results are presented as the mean \pm standard deviation from three independent experiments. ${ }^{\prime} \mathrm{P}<0.05,{ }^{* *} \mathrm{P}<0.01$ vs. the control group; ${ }^{\#} \mathrm{P}<0.05,{ }^{\# \#} \mathrm{P}<0.01$ vs. the cells treated with $\mathrm{NC}$. NC, nitidine chloride; ERK, extracellular-signal-regulated kinase; U0, U0126; cpm, counts per minute.

Inhibition of the ERK pathway enhances the pro-apoptotic and anti-proliferative effects of NC in CRC cells. To further investigate whether the effects of $\mathrm{NC}$ are ERK-dependent, U0126 (MEK1/2 inhibitor which are upstream of ERK, therefore results in ERK inhibition) was used to prevent ERK activation. As shown in Fig. 4C and D, inhibition of ERK activity using U0126 enhanced the upregulation of Bax, cleaved caspase- 3 and -9 expression and the downregulation 
of Bcl-2 expression induced by NC. This demonstrated that NC-induced apoptosis may be ERK dependent. To further investigate the anti-proliferative effects of $\mathrm{NC}$, the proliferation of CRC cells was measured using an MTT assay. As shown in Fig. 5A, the ERK inhibitor significantly enhanced the NC-induced inhibition of CRC cell proliferation. Similar results were observed using $\left[{ }^{3} \mathrm{H}\right]$ thymidine uptake (Fig. 5B), which demonstrated that preventing ERK activation was able to significantly enhance the NC-induced inhibition of CRC cell proliferation.

\section{Discussion}

Uncontrolled proliferation and/or resistance to apoptosis, provides cancer cells with a survival advantage to resist conventional chemotherapeutic agents $(15,16)$. In addition, conventional chemotherapeutic drugs are limited for long-term use due to their toxicity and side-effects on normal cells. In recent years, increasing attention has been focused on the therapeutic functions of natural products in cancer therapy, which have fewer side-effects (17-19). As a natural, bioactive phytochemical alkaloid extracted from Zanthoxylum nitidum (Roxb), NC has exhibited a wide range of pharmacological activity against inflammation and oxidation $(6,7)$. In recent years, increasing evidence suggests that $\mathrm{NC}$ has the ability to induce apoptosis and/or inhibit the proliferation, migration and invasion of renal, breast, hepatocellular and gastric cancer cells (8-13). Therefore, NC is a promising chemotherapeutic agent for various types of cancer. However, although previous studies have shed light on the antitumor activity of $\mathrm{NC}$, whether $\mathrm{NC}$ has any effect on the proliferation and apoptosis of CRC cells remains unknown, as do the detailed molecular mechanisms involved. The current study, to the best of our knowledge, was the first demonstration that $\mathrm{NC}$ is able to inhibit proliferation and induce apoptosis in CRC cells. In addition, $\mathrm{NC}$ was demonstrated to have this effect on CRC cells via the ERK signaling pathway. These data provide a novel molecular mechanism by which NC exerts its anti-cancer effect in CRC cells.

Apoptosis is regulated by pro-apoptotic and anti-apoptotic proteins, and is executed through caspases (20). The balance of Bcl-2 protein family members, including anti-apoptotic proteins (Bcl-2, Bcl-xL) and pro-apoptotic proteins (Bax, Bad), serves a crucial role in modulating and executing a number of apoptotic pathways $(21,22)$. In previous studies, the anti-apoptotic Bcl-2 protein has been shown to function as a preserver of the mitochondrial membrane, preventing the release of internal calcium into the cytoplasm and inhibiting the oligomerization of the anti-apoptotic Bax protein $(23,24)$. Bax has been shown to translocate into mitochondria to regulate cytochrome $\mathrm{C}$ release, thus resulting in the activation of caspase-3 and -9 , and inducing apoptosis $(25,26)$.

As reported in previous studies $(8,11)$, NC exhibits potent activity in inhibiting the proliferation of cancer cells by inducing apoptosis and modulating the expression of the $\mathrm{Bcl}-2$ family. In the present study, $\mathrm{NC}$ was observed to induce apoptosis and inhibit proliferation in CRC cells, in a doseand time-dependent manner. Furthermore, the expression of the pro-apoptotic protein, Bax, and the anti-apoptotic protein, $\mathrm{Bcl}-2$, was measured in CRC cells treated with NC. NC was observed to upregulate the expression of Bax and downregulate the expression of Bcl-2. Additionally, caspase-3 and -9 were further activated by $\mathrm{NC}$ treatment, resulting in apoptosis. These results suggest that NC-induced CRC cell apoptosis may be attributed to the reduced expression of $\mathrm{Bcl}-2$ and the increased expression of Bax, resulting in the activation of caspase-3 and -9 . A previous study demonstrated that the role of the tumor suppressor p53 in apoptosis was associated with several Bcl-2 family members (27). In the present study, p53 expression in CRC cells was measured following treatment with NC, which indicated that NC upregulated the expression of p53 in a dose-dependent manner. This result further demonstrates that p53 was activated in the NC-induced apoptosis of CRC cells.

As a key signal transduction pathway, the ERK signaling pathway is involved in the regulation of proliferation, differentiation, senescence and apoptosis in cancer cells (28). Previous studies have revealed that cell survival and apoptosis are modulated by the ERK signaling pathway $(29,30)$. In the present study, the ERK activity in CRC cells was detected by western blot analysis. The results showed that the expression of p-ERK was reduced following treatment with NC. To confirm that the ERK signaling pathway was involved in the NC-induced apoptosis and the inhibition of proliferation in CRC cells, ERK phosphorylation was prevented using U0126, the inhibitor of MEK1/2, which are upstream of ERK, therefore resulting in ERK inhibition.. This indicated that the inhibition of ERK activity by U0126 enhanced the upregulation of Bax, caspase- 3 and -9 , and the downregulation of Bcl-2 induced by NC. In addition, ERK inhibition resulted in the inhibition of cell proliferation. These results suggest that NC induced the apoptosis of CRC cells through the suppression of ERK activity, and by altering the expression of Bax and Bcl-2. Furthermore, ERK inhibition by U0126 enhanced the increased expression of Bax and reduced expression of Bcl-2 induced by $\mathrm{NC}$, further indicating that ERK was upstream of Bax and Bcl-2.

In conclusion, the present study demonstrated that $\mathrm{NC}$ inhibits the proliferation of CRC cells and induces apoptosis. Furthermore, the effect of NC was mediated through the ERK signaling pathway. Therefore, the current study suggests that $\mathrm{NC}$ is a potential therapeutic agent for the treatment of colorectal cancer. Further in vivo studies should be performed to confirm these results and investigate the effects further.

\section{Acknowledgements}

The current study was supported by the Natural Science Foundation of China (grant no. 81370325) and the Yantai Scientific Development Project (grant no. 2013WS216).

\section{References}

1. Jemal A, Bray F, Center MM, Ferlay J, Ward E and Forman D: Global cancer statistics. CA Cancer J Clin 61: 69-90, 2011.

2. Center MM, Jemal A, Smith RA and Ward E: Worldwide variations in colorectal cancer. CA Cancer J Clin 59: 366-378, 2009.

3. Cunningham D, Atkin W, Lenz HJ, Lynch HT, Minsky B, Nordlinger B and Starling N: Colorectal cancer. Lancet 375: 1030-1047, 2010. 
4. Jiang WQ, Fu FF, Li YX, Wang WB, Wang HH, Jiang HP and Teng LS: Molecular biomarkers of colorectal cancer: Prognostic and predictive tools for clinical practice. J Zhejiang Univ Sci B 13: 663-675, 2012.

5. Gordaliza M: Natural products as leads to anticancer drugs. Clin Transl Oncol 9: 767-776, 2007.

6. Wang Z, Jiang W, Zhang Z, Qian M and Du B: Nitidine chloride inhibits LPS-induced inflammatory cytokines production via MAPK and NF-kappaB pathway in raw 264.7 cells. J Ethnopharmacol 144: 145-150, 2012.

7. Del Poeta M, Chen SF, Von Hoff D, Dykstra CC, Wani MC, Manikumar G, Heitman J, Wall ME and Perfect JR Comparison of in vitro activities of camptothecin and nitidine derivatives against fungal and cancer cells. Antimicrob Agents Chemother 43: 2862-2868, 1999.

8. Fang Z, Tang Y, Jiao W, Xing Z, Guo Z, Wang W, Xu Z and Liu Z: Nitidine chloride induces apoptosis and inhibits tumor cell proliferation via suppressing ERK signaling pathway in renal cancer. Food Chem Toxicol 66: 210-216, 2014

9. Fang Z, Tang Y, Jiao W, Xing Z, Guo Z, Wang W, Shi B, Xu Z and Liu Z: Nitidine chloride inhibits renal cancer cell metastasis via suppressing AKT signaling pathway. Food Chem Toxicol 60: 246-251, 2013.

10. Pan X, Han H, Wang L, Yang L, Li R, Li Z, Liu J,Zhao Q, Qian M, Liu M and Du B: Nitidine chloride inhibits breast cancer cells migration and invasion by suppressing c-Src/FAK associated signaling pathway. Cancer Lett 313: 181-191, 2011.

11. Sun M, Zhang N, Wang X, Cai C, Cun J, Li Y, Lv S and Yang Q: Nitidine chloride induces apoptosis, cell cycle arrest, and synergistic cytotoxicity with doxorubicin in breast cancer cells Tumour Biol 35: 10201-10212, 2014.

12. Liao J, Xu T, Zheng JX, Lin JM, Cai QY, Yu DB and Peng J: Nitidine chloride inhibits hepatocellular carcinoma cell growth in vivo through the suppression of the JAK1/STAT3 signaling pathway. Int J Mol Med 32: 79-84, 2013.

13. Chen J, Wang J, Lin L, He L, Wu Y, Zhang L, Yi Z, Chen Y, Pang $X$ and Liu M: Inhibition of STAT3 signaling pathway by nitidine chloride suppressed the angiogenesis and growth of human gastric cancer. Mol Cancer Ther 11: 277-287, 2012.

14. Sebolt-Leopold JS and English JM: Mechanisms of drug inhibition of signalling molecules. Nature 441: 457-462, 2006.

15. Adams JM and Cory S: The Bcl-2 apoptotic switch in cancer development and therapy. Oncogene 26: 1324-1337, 2007.

16. Cai Q, Lin J, Wei L, Zhang L, Wang L, Zhan Y, Zeng J, Xu W, Shen A, Hong Z and Peng J: Hedyotis diffusa Willd inhibits colorectal cancer growth in vivo via inhibition of STAT3 signaling pathway. Int J Mol Sci 13: 6117-6128, 2012.
17. Surh YJ: Cancer chemoprevention with dietary phytochemicals Nat Rev Cancer 3: 768-780, 2003.

18. Tan AC, Konczak I, Sze DM and Ramzan I: Molecular pathways for cancer chemoprevention by dietary phytochemicals. Nutr Cancer 63: 495-505, 2011.

19. Thomasset SC, Berry DP, Garcea G, Marczylo T, Steward WP and Gescher AJ: Dietary polyphenolic phytochemicals-promising cancer chemopreventive agents in humans? A review of their clinical properties. Int J Cancer 120: 451-458, 2007.

20. Zhang Y, Zhuang Z, Meng Q, Jiao Y, Xu J and Fan S: Polydatin inhibits growth of lung cancer cells by inducing apoptosis and causing cell cycle arrest. Oncology Lett 7: 295-301, 2014.

21. Reed JC: Bcl-2: Prevention of apoptosis as a mechanism of drug resistance. Hematol Oncol Clin North Am 9: 451-473, 1995

22. Brady HJ and Gil-Gómez G: Bax. The pro-apoptotic Bcl-2 family member, Bax. Int J Biochem Cell Biol 30: 647-650, 1998.

23. Baffy G, Miyashita T, Williamson JR and Reed JC: Apoptosis induced by withdrawal of interleukin-3 (IL-3) from an IL-3-dependent hematopoietic cell line is associated with repartitioning of intracellular calcium and is blocked by enforced $\mathrm{Bcl}-2$ oncoprotein production. J Biol Chem 268: 6511-6519, 1993.

24. Precht TA, Phelps RA, Linseman DA, Butts BD, Le SS, Laessig TA, Bouchard RJ and Heidenreich KA: The permeability transition pore triggers Bax translocation to mitochondria during neuronal apoptosis. Cell Death Differ 12: 255-265, 2005.

25. Antonsson B: Bax and other pro-apoptotic Bcl-2 family 'killer-proteins' and their victim the mitochondrion. Cell Tissue Res 306: 347-361, 2001.

26. Crompton M: Bax, Bid and the permeabilization of the mitochondrial outer membrane in apoptosis. Curr Opin Cell Biol 12: 414-419, 2000.

27. Yu Q: Restoring p53-mediated apoptosis in cancer cells: New opportunities for cancer therapy. Drug Resist Updat 9: 19-25, 2006.

28. Thompson $\mathrm{N}$ and Lyons $\mathrm{J}$ : Recent progress in targeting the Raf/MEK/ERK pathway with inhibitors in cancer drug discovery. Curr Opin Pharmacol 5: 350-356, 2005.

29. Gendron S, Couture J and Aoudjit F: Integrin alpha2beta1 inhibits Fas-mediated apoptosis in T lymphocytes by protein phosphatase 2A-dependent activation of the MAPK/ERK pathway. J Biol Chem 278: 48633-48643, 2003.

30. Shelton JG, Steelman LS, White ER and McCubrey JA: Synergy between PI3K/Akt and Raf/MEK/ERK pathways in IGF-1R mediated cell cycle progression and prevention of apoptosis in hematopoietic cells. Cell Cycle 3: 372-379, 2004. 